\title{
Design, implementation and application of an intelligent system for territorial risks assessment
}

\author{
Ulyana S. Postnikova ${ }^{1}$, Valeriy V. Nicheporchuk ${ }^{2}$ and Olga V. Taseiko ${ }^{1,3}$ \\ ${ }^{1}$ Krasnoyarsk Branch of the Federal Research Center for Information and Computational Technologies, Krasnoyarsk, \\ Russia \\ ${ }^{2}$ Institute of Computational Modeling SB RAS, Krasnoyarsk, Russia \\ ${ }^{3}$ Reshetnev Siberian State University of Science and Technology, Krasnoyarsk, Russia
}

\begin{abstract}
The process describes an intelligent system creation designed for risk evaluation and management. The risk can be anthropogenic, natural, or social nature, and belong to territories of different scales. Complexity in structuring and collecting information about the state territorial security as well as different risks assessment methods necessitate the development of a modular multitask system. The information management system support model formalizes the problem area to justify the joint intelligent technologies use. Based on the model, system architecture has been developed. This architecture defines the composition, functionality, interaction interfaces, as well as the information resources organization, that were used to support management. Here is presented an intelligent system prototype operation result.
\end{abstract}

\section{Keywords}

Model system, intelligent technologies, system architecture, structure of information resources, assessment and management of territories risks.

\section{Introduction}

Maintaining the territorial safety is a challenging task, especially when the economy fails. However, existing technologies readily provide an opportunity to implement low-cost measures allowing to manage social, natural, and technogenic risks. It is important to study various territorial characteristics, treating them as complex systems, in order to find and justify these measures. One of the most theoretically well-supported concepts is the idea of the territory as a complex socio-natural-technogenic system where multiple risk groups are formed and implemented [1]. The construction of an intelligent risk assessment system should be based on formalizing a sufficient number of socio-economic and natural-climatic indicators, integrated assessment algorithms, data processing and visualization, and the coordination of systemic and external services.

In order to establish acceptable various risks' levels for conducting a sociological survey, a risk classification was carried out as part of the iNTeg-Risk project [2]. Data was collected and processed to assess and analyze risks as well as their acceptable levels and methods of managing

SDM-2021: All-Russian conference, August 24-27, 2021, Novosibirsk, Russia

Ðulyana-ivanova@inbox.ru (U.S. Postnikova); valera@icm.krasn.ru (V.V. Nicheporchuk); taseiko@gmail.com

(O. V. Taseiko)

(c) (1) $\odot 2021$ Copyright for this paper by its authors. Use permitted under Creative Commons License Attribution 4.0 International (CC BY 4.0).

CEUR Workshop Proceedings (CEUR-WS.org) 
them. The developed systems of analysis (NETworked) and risk management (NET-HARMS) are able to identify and predict systemic and emerging risks that occurred in accidents described by data sets.

To control territories in the EU countries, the concept of "smart city" or "smart territory" based on the criteria for sustainable and effective development is actively being developed. This concept covers various spheres of life: energy, transport infrastructure, resource consumption, environmental impact, etc. It requires a detailed analysis and diagnosis of the territory and key indicators to create such a system [3]. The concept of a "smart city" has a strong theoretical foundation; however, in reality it works only in isolation, and does not consider any risks thereby losing the most of its value.

The results obtained by the researches $[4,5,6,7]$ prove the necessity of data integration, taken from incidents and formalized industrial safety indicators. Public safety requirements have increased recently, especially in the light of global events and trends related to climate change, digitalization of private data, the COVID-19 pandemic, as well as constant growth and development of technical systems and industry. However, periodically occurring disasters show the failure of efforts to prevent them, alongside with the necessity to consolidate large monitoring data and utilization of intelligent technologies to search for a new knowledge $[8,9,10]$.

This work proposes the "end-to-end" technology creation for designing and building an intellectual system responsible for assessing territorial risks. It briefly describes the contents of the informational support systemic model for territorial risk management, which underlies the architecture of the intellectual system. The presented architecture allows you to create multitask problem-oriented systems; some of them are already implemented in the Siberian region.

\section{Model of information support management}

The result of the first stage building an intelligent system is a management support system model $\mathrm{M}$ that formalizes the basic requirements, composition and elements interaction in the form [11]:

$$
M=\langle G, T, L, F, C, I T, Y\rangle,
$$

where $G$ - management objectives; $T$ - control tasks; $L$ - decision levels; $F$ - system functions; $C-$ containers integrating information resources, their processing methods and characteristics for each type of risk; IT - information technologies; $Y$ - formed decisions. The listed elements of the model are detailed as sets.

Management goals set $G=\left\{g_{1}, g_{2}, g_{3}\right\}$ include: $g_{1}$ - increase life expectancy by reducing the number of dead and deceased prematurely; $g_{2}$ - increase in the healthy life duration by reducing the number of people who need treatment, rehabilitation and social protection; $g_{3}$ - comfortable living environment creation, including the environment normalization and uninterrupted resources supply. All management goals have a multiplier effect - financial investments in preventive measures are significantly less than the cost of unprevented harm [12]. Tasks set $T=\left\{t_{1}, t_{2}, t_{3}\right\}$ are grouped by their implementation frequency and include the following: $t_{1}$ - the risk of reduction measures that are performed continuously; $t_{2}-$ seasonal preventive measures peculiar to cyclical emergencies, environmental, anti-epidemic and other 
measures; $t_{3}$ - one-time events that radically reduce the level of risk. Strategic decisions on the territorial security management that require collection and processing large amounts of data are made at the regional level $l_{1}$. Municipal $l_{2}$ and object $l_{3}$ levels have limited functions when it comes to collecting and viewing data. Such a division is reflected in the intelligent system architecture which provides different human-machine interfaces. The set of functions $F=\left\{f_{1}, f_{2}, f_{3}, f_{4}\right\}$, where $f_{1}$ is the collection and consolidation of data; $f_{2}$ - analytical data processing and calculations; $f_{3}-$ dynamic visualization of results; $f_{4}$ - decisions making.

Containers in an intelligent system are presented as pairs $C=\{D, A\}$, where $D$-information resources, $A$ - methods and algorithms that process and present data using information technologies $I T[13,14]$. In its turn, $I T=\left\{i t_{1}, \ldots, i t_{5}\right\}$, where $i t_{1}-$ data warehouse technologies; $i t_{2}$ - analytical data processing technologies; $i t_{3}$ - intelligent technologies; $i t_{4}-$ geoinformation technologies; $i t_{5}$ - web technologies. Information resources $D=\left\{d_{1}, \ldots, d_{5}\right\}$, where $d_{1}$ - system-forming elements (reference books, classifiers); $d_{2}$ - monitoring processes (events) data; $d_{3}$ - analyzed objects characteristics; $d_{4}$ - spatial data; $d_{5}$ - knowledge bases. The typical structure of $D$ elements is developed using UML notations. Based on the structure of information resources description, the intellectual system data warehouse was designed and filled.

Let us focus on the description of knowledge bases. Their structure in a generalized form reflects the representation of a multilevel aggregated knowledge model used in the solving control problem process and described in [15]. Knowledge bases are represented by the set $d_{5}=\langle$ frames, rules, models, solver, interfaces, thesaurus, decisions $\rangle$, where frames - the base of frames representing possible scenarios for changing the analyzed objects characteristics; rules - a rule base "condition-action-rules" type; models - calculation library models; solver inference machine; interfaces - knowledge library base interfaces; thesaurus - a dictionary describing fact variables and their properties; decisions - decision projects database. Frames form a scenario for the risk reduction measures behavior. Rules represent actions and conditions for their execution and are used as attached procedures in frames. Models are needed to assess the consequences of activities and describe the new state of security for a certain territory. The solver interprets the calculation and interface procedures, implements the withdrawal strategy by selecting the preferred rule or procedure from the characteristics of the evaluated territory applicable to the array, in accordance with the specified criteria. As a result, "anonymous" decision templates are filled with specific information about the types, sequence and expected outcomes. The implementation of the $d_{5}$ structure in the repository is presented as logically related tables.

Integrating these technologies in control systems allows synthesizing solutions that coordinate the actions of experts from various departments involved in the targeted risk reduction programs development. Updated information resources increase the decision effectiveness when it comes to working with lack of time, the presence of incomplete and obscure initial information, and the error high costs.

The intelligent system work results largely depend on the volume and content of the data, which is different for different territories. The output can be represented as $Y=\{B, H, Q, K\}$, where $B$ - text recommendations and explanations; $H$ - tables; $Q$ - graphical representations; $K$ - dynamic maps visualizing the territorial risks distribution. For a better understanding, $B$ includes the extensive terminology used in risks assessment $[16,17]$. 
Elements from sets of this system model can be supplemented with the new data processing technologies advent, information resources types, as well as territorial management tasks.

\section{A conceptual description of the architecture of an intelligent system}

The next step in the intelligent system implementation is architectural design, linking the system model elements, and the information processes decomposition of the system's interaction with the external environment, and the program modules functioning within the system.

The multitasking problem-oriented intelligent system architecture is based on the system management model. Figure 1 shows context diagram of the system interacting with the environment. This configuration allows getting results when performing various combinations of functions $F$. For example, $f_{1}$ data collection results and consolidation are available to other systems through a data gateway. Performing risk assessments (functions $f_{2}, f_{3}$ ) without forming solutions $f_{4}$ is advisable in case of information resources deficiency.

Using the control support model elements, control tasks $T$ were decomposed as functional diagrams. This allowed describing in detail the transformation processes of information resources using IT technologies. The architecture concretizes the intelligent system design describing a unit of consolidating information resources, subsystems and data processing services, and human-machine interfaces (Figure 2).

Consolidation processes are described in accordance with the classification of information resources and information technologies introduced in the system model. The use of systemforming resources $\mathrm{d} 1$ to enrich monitoring data during the consolidation process in the repository made it possible to implement various analytical processing technologies, such as POD (post OLAP dynamics), Data Mining, and others $[18,19]$. The main information resources array was used to assess territorial risks is data from monitoring processes and events $d_{2}$, as well as analyzed objects $d_{3}$ characteristics. The basic spatial data $d_{4}$ is used for the formation of cartograms and overview maps is placed in the repository. Detailed cartographic territorial descriptions are available through WMS and other services described in [20]. To collect formalized management processes in $d_{5}$ knowledge bases, a graphical method proposed for displaying a sequence of "elementary" operations, similar to IDEFx notation.

Due to significant differences in the data structure, processing and presentation result methods, it is advisable to integrate them for each risk type by the type of container processing. The

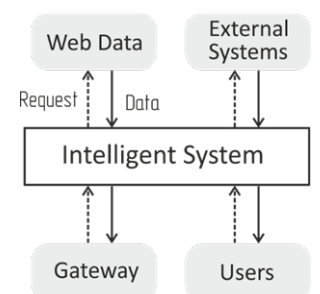

Figure 1: Context diagram of an intelligent system. 


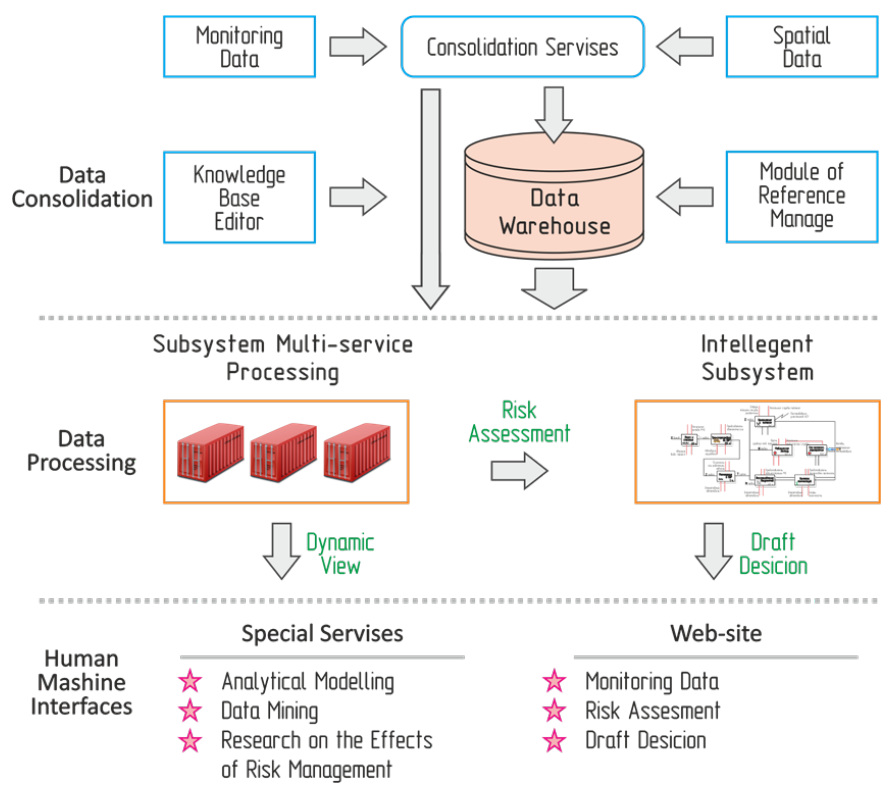

Figure 2: The architecture of an intelligent system.

growth of intellectualization system is possible with the mass knowledge bases formation that describes the preventive measures management processes. A graphical interface is being developed to transform formalized descriptions of multi-step actions. The large training sample formation makes it possible to use neural networks and other intelligent technologies. This allows creating several alternative solutions with ranking them by priority.

Human-machine interfaces are designed taking into account different decision-making levels $L$. Different dynamic representations of the processing results (elements of the set $Y$ ) allow avoiding the reduction of risk assessments as a single numerical indicator. The support includes various access mechanisms for individuals who form and make managerial decisions - desktop software systems, websites, and mobile applications.

The developed architecture made it possible to determine the synthesized intelligent system functionality and substantiate the software components and rational methods choice for the integrated control problems solution.

\section{Assessment of territorial risks in the intelligent system}

To assess territorial risks, it is proposed to collect and analyze monitoring data and statistics on emergencies and dangerous events, including industrial safety violations, public health, and environmental monitoring data. The proposed approach difference is using formalized characteristics of the danger sources, objects' vulnerability, and the state of protection systems [21].

The territorial risks assessment takes place in three main stages:

- definition and formation of a source data array;

- risks calculations and analytical modeling; 
- analysis results dynamic presentation, including risks mapping.

During the first stage, there are determined possible dangers for the territories, initial data, general target information and models for the analytical risks study are formed. When performing the second stage, it is necessary to divide the risks into two fundamental groups, characterized by the exposure duration (instant and long-term effect). At the third stage, recommendations are developed for risk management at the municipal level.

The risk calculation is carried out in order to determine the necessity and effectiveness of preventive measures, as well as measures to manage the municipality's developmental risks by executive authorities.

\section{Conclusions}

The architecture's basic functions are implemented in the integrated management support systems "ESLA-PRO" and the Risk Analysis System SAR ES. Operational experience has shown the necessity to improve intersystem information exchange using distributed ledger technologies, cloud data storage, consolidating information from industry systems. Also have been created updated regional atlases of emergency situations covering natural and anthropogenic natures, some of them are published on the ICM SB RAS [21] geographic informational portal.

The proposed creation method of an intelligent system for assessing and managing social, natural and anthropogenic systems' risks allows building up a comprehensive information platform for solving a wide range of territorial management tasks. The method is based on the management information support model and the intelligent system architecture that substantiate the original integration of information resources and technologies. The combination of an intelligent system with integrated monitoring services will systematically reduce the human and social life risks, as well as the ecological systems' state to acceptable values that are currently achieved in only a small number of countries [22].

\section{Acknowledgments}

This research project No. 18-47-240006: "Methods and information technologies for risk assessment of the development of socio-natural-technogenic systems in an industrial region" was funded by the Russian Foundation for Basic Research, Government of Krasnoyarsk Territory, Krasnoyarsk Regional Fund of Science.

\section{References}

[1] Dallat C., Salmon P.M., Goode N. Identifying risks and emergent risks across sociotechnical systems: The NETworked hazard analysis and risk management system (NETHARMS) // Theoretical Issues in Ergonomics Science. 2018. Vol. 19. N. 4. P. 456-482. DOI:10.1080/1463922X.2017.1381197.

[2] Neirotti P. Current trends in Smart City initiatives: Some stylised facts // Cities. 2014. Vol. 38. P. 25-36. DOI:10.1016/j.cities.2013.12.010. 
[3] Scheer D. Risk governance and emerging technologies: Learning from case study integration // Journal of Risk Research. 2013. Vol. 16. N. 3-4. P. 355-368. DOI:10.1080/13669877.2012.729519.

[4] Knijff P., Allford L., Schmelzer P. Process safety leading indicators. A perspective from Europe // Process Safety Progress. 2013. Vol. 32. N. 4. P. 332-336.

[5] Leveson N. A systems approach to risk management through leading safety indicators // Reliability Engineering \& System Safety. 2015. Vol. 136. P. 17-34.

[6] Øien K., Utne I., Tinmannsvik R., Massaiu S. Building safety indicators II applications // Safety Science. 2011. Vol. 49. P. 162-171.

[7] Warden P. Big Data Glossary. Sebastopol: O’Reilly Media, Inc., 2011.

[8] Lea P. Internet of Thing for Architects. Birmingham-Mumbai: Packt Publishing, 2018. $454 \mathrm{p}$.

[9] Goodfellow I., Bengio Y., Courville A. Deep Learning. Cambridge; Massa-chusetts; London: The MIT Press, 2017. 653 p.

[10] Kossiakoff A., Sweet W.N., Seymor S.J., Biemer S.M. System engineering principles and practice. John Wiley, 2011. $624 \mathrm{p}$.

[11] Malinetskii G.G., Podlazov A.V., Kuznetsov I.V. On a national scientific monitoring system // Herald of the Russian Academy of Sciences. 2005. Vol. 75. N. 4. P. 323-336.

[12] Negus C., Henry W. Docker containers. Build and deploy with Kubernetes, Flannel, Cockpit, and Atomic. Indiana: Pearson Education, Inc., 2015. 319 p.

[13] Mouat A. Using dockers. Sebastopol: O’Reilly Media Inc., 2015. 328 p.

[14] Nozhenkova L.F. Efficient inference in production systems for data interpretations // Scientific Siberia. Ser. A. Vol. 11, Numerical and Data Analysis. Tassin: AMSE Press, 1994. P. 131-154.

[15] International standard ISO 22300:2018. Security and resilience - Terminology.

[16] PreventionWeb. The knowledge platform for disaster risk reduction. Available at: https: //www.preventionweb.net/Terminology.

[17] Zaki M.J., Wagner M.J. Data mining and machine learning: Fundamental concepts and algorithms. Cambrige Univesity Press, 2020.760 p.

[18] Penkova T., Korobko A., Nicheporchuk V., Nozhenkova L., Metus A. Online control of the natural and anthropogenic safety in Krasnoyarsk Region // International Journal of Social, Behavioral, Educational, Economic and Management Engineering: World Academy of Science, Engineering and Technology, 2015. Vol. 9. N. 8. P. 2336-2341.

[19] Bychkov I.V., Vladimirov D.Ya., Oparin V.N., Potapov V.P., Shokin Yu.I. Mining information science and Big Data concept for integrated safety monitoring in subsoil management // Journal of Mining Science. 2016. Vol. 52. N. 6. P. 1195-1209. DOI:10.1134/S1062739116061747.

[20] Penkova T., Nicheporchuk V., Metus A. Comprehensive operational control of the natural and anthropogenic territory safety based on analytical indicators // Proceedings of the International Joint Conference IJCRS 2017. Olsztyn, Poland, July 2017. Part I. P. 263-270, DOI:10.1007/978-3-319-60837-2.

[21] Geoinformation portal ICM SB RAS. Available at: https://gis.krasn.ru.

[22] Index for risk management. Available at: https://drmkc.jrc.ec.europa.eu/inform-index. 